\title{
Increasing Lifetime through Sleep Awake and Conditional Transmission in Wireless Body Area Networks
}

\author{
https://doi.org/10.3991/ijim.v13i02.9868
}

Karthik. $\mathbf{J}^{(凶)}$

St Peters University, Chennai, Tamil Nadu, India

karthikvalavan@gmail.com

Dr. A. Rajesh

C. Abdul Hakeem College of Engineering and Technology, Vellore, Tamil Nadu, India.

\begin{abstract}
Human health is being monitored by wireless sensors from their home using wireless body area networks. Increase in the wireless body sensors made human to monitor health with great ease. Patient need not be stay in hospital for long time instead they may use body sensors and they may monitor their health from their residence itself. So that patients easily will be moving around their residence. Even though development of technology made ease of every task there are also constraints that need to be reduced. Since the body nodes are very tiny the battery used for the sensor is also small, in turn the battery capacity is also greatly reduced. So the life time of the sensor nodes are very low and in turn network lifetime also will be very less. In order to increase the life time of the node energy consumption should be monitored with more care. In our proposed system we implement sleep awake method along with conditional transmission to reduce the energy consumption that automatically increases the life time of the node as well as network. Our proposed method gives better result when compare with the performance of other methods in saving energy.
\end{abstract}

Keywords—Energy, sleep awake, conditional transmission, network lifetime, body sensors.

\section{Introduction}

Nowadays human health is being affected more by various diseases. So they may need to be stayed in hospital for long time. Very often people are going to hospital. Particularly aged people are suffering by more illness and that may cause to stay in the hospital for long time. People are feeling uncomfortable when they are in the hospital and that situation itself affects human heath badly. Patients are needed to be set freely to recover from illness very quickly. But at the same time they need to be monitored very carefully to ensure that they are not in critical health condition. This can be done with the help of wireless body area sensor networks. 
Wireless sensors are being placed in, on and around the body to monitor the human health at every moment. These sensors monitor the human health and send the report to the medical practitioners. They will suggest the medicine and treatment to recover from the illness and if they needed any care in direct then that can be provided by the practitioners. Even though the effort is being reduced to monitor human health it is mandatory to ensure that the device is properly functioning without any failure.

Since body sensors are very small, the battery used in that sensor is also very tiny. Size of the battery greatly affects the storage capacity of power in it. So sensor batteries are needed to be replaced very often. It is difficult and it may affect the human health. To avoid this problem we need to utilize the energy effectively. Only then the batteries are need not be replaced very frequently. Power consumption plays vital role in the lifetime of sensor node. Mainly more energy will be consumed by the transceiver rather by the processor in the sensor node. So by reducing the number of transmission it is possible to reduce the energy consumption in the sensor node. Sensor nodes are spending more energy by being idle for long time. By keeping node in sleep, state will greatly reduce the energy consumption by avoiding being idle.

Only one sensor node can monitor the human health while all others are in sleep state. Sleeping nodes are can be awaken if needed, that is if any abnormal values are identified by the sensor node then the active node will stimulate all other nodes to monitor the values. So the ideal monitoring will be reduced to save the energy.

With this conditional transmission and sleep awake together the energy consumption will be reduced to a great extent. Thus, the body nodes lifetime will be increased and replacement of batteries can be reduced. Therefore the life time of the network is also increased. The discussion about the previous papers and the existing energy consumption reduction methods are explained in chapter II. The proposed method sleep awake concept and conditional transmission technique is explained in chapter III and chapter IV. The sample results are discussed in chapter $\mathrm{V}$ followed by the conclusion and future enhancement in chapter VI. The further detailed information provided in following chapters.

\section{Literature Survey}

In general all sensor nodes have memory to store, processor to process the data, battery to supply the power to the sensor components, Sensing device to sense the values and communication device to transmit and to receive the data [1]. More energy is consumed by the major three activities such as sensing, calculation and transmission in any sensor device. Particularly transmission takes more energy than any activity in a sensor node. Energy loss is more by the transceiver device since the device sends and receives signal very frequently [2].

Color based topology control algorithm were used to reduce the energy consumption of wireless sensor nodes [3]. By reducing the activity of transceiver, prolong the lifetime of the node. They introduced adaptive sleep protocol, which adjusts sleep schedule of any sensor nodes [4]. From Praveen kaushik [5], efficient routing algorithm can help to increase the lifetime by reducing the energy consumption and also they proposed 
sleep schedule algorithm. Sensors nodes are need to power down unused or few components of the node or the whole node to reduce the energy consumption. Energy management is also achieved by reducing the transmission and using the computation properly [6]. It is also stated clearly that even though processor needs power while doing some process, it is not consuming as much of energy consumed by transmitting or receiving data.

Duty cycling and energy efficient MAC protocols were discussed by Zahra Rezaei[7], from various MAC protocols energy can be utilized effectively and also various MAC techniques that helps in improving the life time of the sensor node. From [8] cluster head can be formed and that cluster head may communicate with each other, and cluster head can be chosen based on the energy which node has remaining energy.

The distance between two nodes will help to decide the sleeping possibility of the node. Based on this the frequency of the data transmission will be reduced. So the energy consumption can be reduced with the help of finding the distance between the data transmission nodes [9]. According to Luiz [10] Transmission Power Control Techniques can be applied to adjust transmission power. They consider distance between nodes, concurrent transmission and the node mobility.

The various sensors that are used in Wireless Body Area Network (WBAN) were discussed in [11]. They have elucidated the different sensors such as wearable sensors; implant sensors and application of sensors are also explained in brief. The benefits of alternative for two-way transmission to minimize the energy consumption were demonstrated in [12]. They were expresses the posture information and channel relay information plays important role in consuming energy.

Various design methods that helps for designing a model is discussed in [13]. The mobile health care applications were created and implemented for various health issues [14][15].

\section{Sleep Awake Method}

This sleep awake method makes all the sensor nodes to sleep except one or two nodes. Those active nodes are alone monitoring the human health. If it finds any abnormal value that is sensed value is below or above acceptable level then those nodes stimulate all other nodes to sense the value and to send that value to medical practitioner as shown in figure 1.

So that all nodes are needed not to be active state, rather it may keep in sleep state. Due to this the energy consumption will be reduced greatly. Sleep Awake concept can be achieved through many methods like random sleep awake, Synchronized sleep awak demand sleep awake, and so on. In this paper we used on demand sleep awake approach, because sensor nodes are being stimulated only when there is demand. Otherwise all other nodes are in sleep state instead of being in an idle state. This sleep awake concept is represented through algorithm 1. 


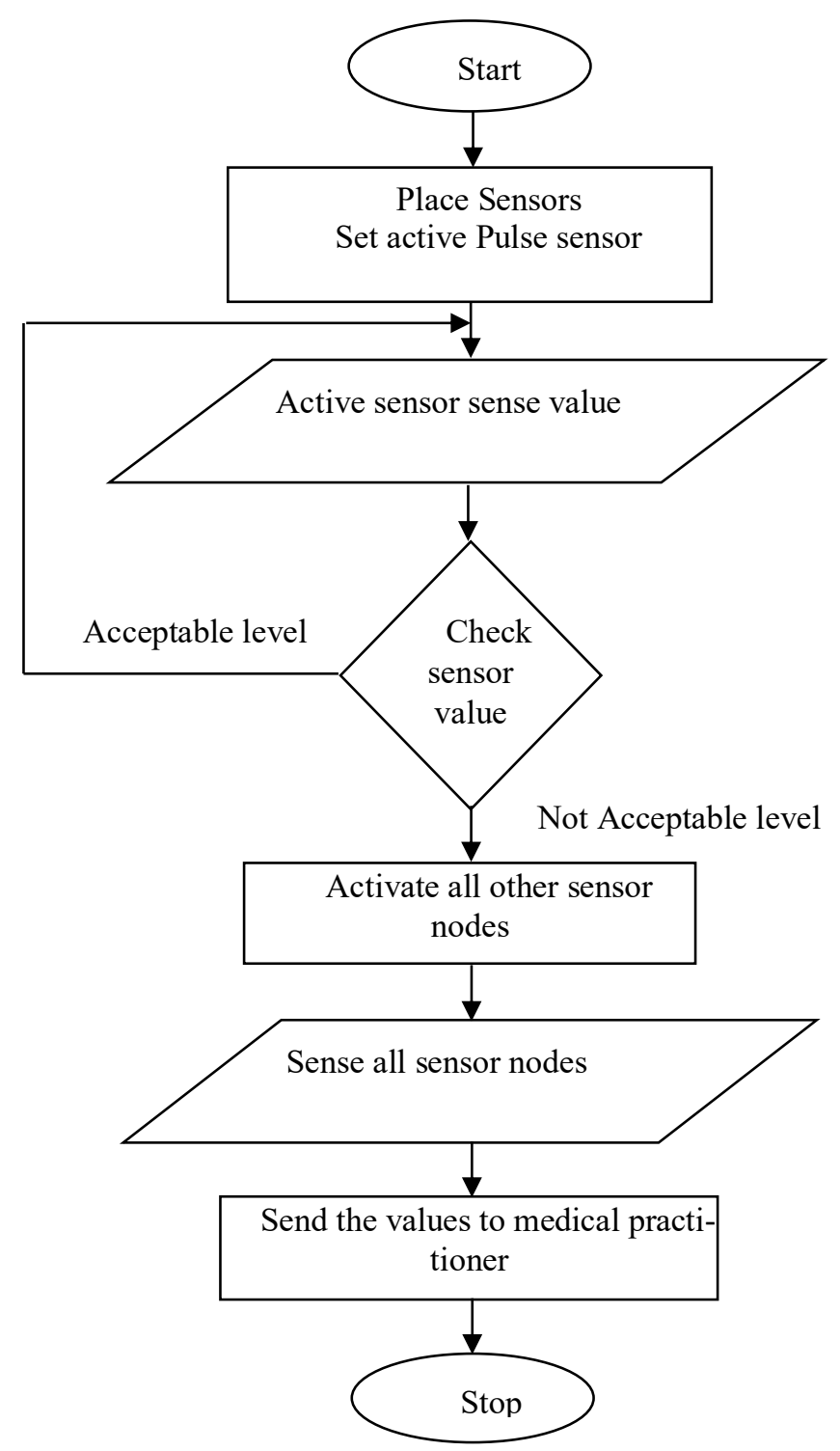

Fig. 1. Flowchart representing sleep awake 
Algorithm 1: On Demand Sleep Awake Algorithm

Step 1: start the process.

Step 2: identify what are the sensors are needed to be fixed.

Step3: let sensor n1, n2 through nk.

Step4: consider $\mathrm{n} 1$ as pulse sensor, and let $\mathrm{n} 1$ be active.

Step5: sense the value by sensor $\mathrm{n} 1$.

Step6: compare the sensed value with threshold value.

Step7: if sensed value by $n 1$ is below or greater than acceptable level then go to step 8 else continue from step5 again.

Step8: stimulate all other nodes from $\mathrm{n} 2$ through nk by sensor $\mathrm{n} 1$.

Step9: all sensors are active and ready to sense the values.

Step10: sensed values by all sensor nodes are sent to access point.

Step11: then necessary action can be taken by medical practitioners.

Step12: stop the process.

This on demand sleep awake algorithm helps to make the sensor nodes to reduce the energy consumption by keeping sensor nodes in sleep state. Because sensor nodes are do not consume energy when they are in sleep state, even in idle state it may consume some amount of energy, but in sleep state it does not consume the energy. The energy consumption of various components in sensor node is given in table1. From table1, it is clear that the components are needed to be sleep state to reduce the energy consumption. The components of sensor node are consuming more energy when it is active state.

Energy consumption of sensor node can be calculated from the below statement.

- Energy $_{\text {sensor }}=\mathrm{K}\left(\left(\right.\right.$ Voltage* Current $^{*}$ Time $\left.)+\mathrm{E}_{\text {on }}+\mathrm{E}_{\text {off }}\right)$

Where

- $\mathrm{K}$ - Number of times sensor device is turn on

- Eon- Energy taken to turn on the device

- Eoff - Energy taken to turn off the device

When the device is in sleep state then the energy consumption will be greatly reduced from the above method, at the same time the number time awakening the node must be less, otherwise it may lead much energy consumption since it is being turn on very often. Energy needed for turn on the device and turn off the device is also considered and, energy is calculated based on the power supply to the device with respect to time. So while keep on running the sensor device, it may need to supply power continuously and in turn it leads for wastage of energy.

It is clear that sensor node spending more energy when it is transmitting and also it spend when it is being idle for long time. So instead of being idle it can be kept in sleep state and when there is need that time it can be awaken to sense the value. 
Table 1. Energy Consumed by individual Sensor Components

\begin{tabular}{|l|l|c|}
\hline \multicolumn{1}{|c|}{ Components } & \multicolumn{1}{c|}{ State } & Values (units) \\
\hline \multirow{2}{*}{ Microprocessor } & Active & $6 \mathrm{~mA}$ \\
\cline { 2 - 3 } & Sleep & $8 \mathrm{~mA}$ \\
\hline \multirow{2}{*}{ Radio Transceiver } & Active & $20 \mathrm{~mA}$ \\
\cline { 2 - 3 } & Sleep & $2 \mathrm{~mA}$ \\
\hline \multirow{2}{*}{ Memory } & Active & $19 \mathrm{~mA}$ \\
\cline { 2 - 3 } & Sleep & $2 \mathrm{~mA}$ \\
\hline \multirow{2}{*}{ Sensor Board } & Active & $5 \mathrm{~mA}$ \\
\cline { 2 - 3 } & Sleep & $2 \mathrm{~mA}$ \\
\hline
\end{tabular}

\section{Conditional Transmission}

From table 1 we can easily identify that sensor node uses more power for transmitting than computing. The wireless sensor node needs to spend more energy by transceiver than controller. Based on this statement node can spend energy for calculating rather transmitting the data. Therefore, it takes only lesser energy for calculating purpose while compare with transmission.

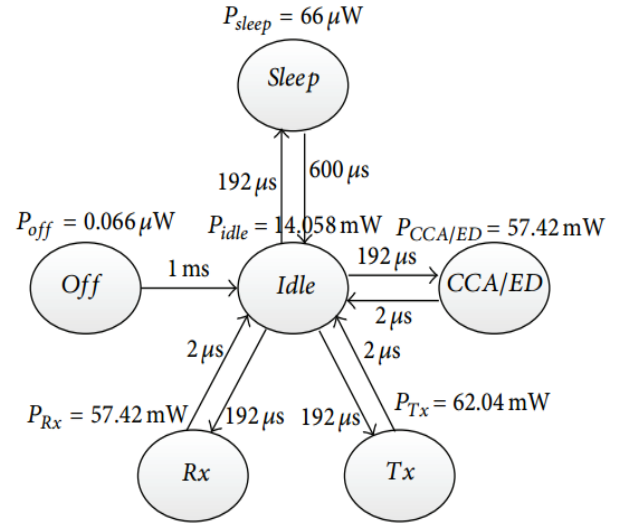

Transceiver CC2420

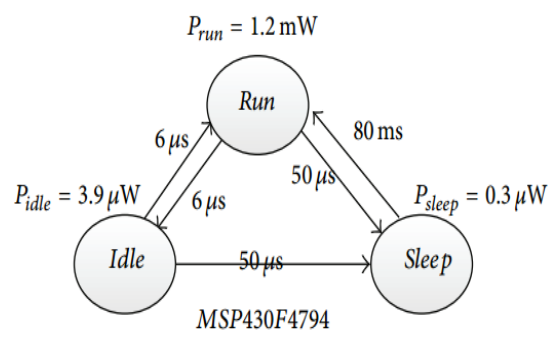

Microcontroller MSP430F4794

Fig. 2. Performance analysis of state transition in WSN node

The node initially calculate the sensed value with the trust value, if the value is abnormal that is above or below threshold value then the node can immediately send the values to the practitioner through access point, until that it may check the sensed value in the node itself. This approach helps to reduce the energy consumption and increase the life time of the node. For this conditional transmission technique we proposed the following algorithm. 
Algorithm 2: Conditional Transmission Algorithm

1: Conditional Transmission

2: let the sensor node be in active state

3: consider Svalue be the value from the sensor

4: compare this Svalue with the threshold value

5 : if (Svalue is not in the range of threshold value) then

6: $\quad$ Awake all other sensor node

7: $\quad$ Send the sensed value to practitioner

8: else

9: $\quad$ continue step 3

10: end if

The sensor node will consume more energy when the sensed value is changing very often. Because the node has to be change its state from idle to active if the value is above or below threshold value. Threshold value refers to acceptable value of pulse sensor.

\section{$5 \quad$ Results and Discussion}

From figure 2 it is clear that the data transmission will consume more energy than calculation. In normal transmission (ie) without conditional transmission the number of transmissions is high. So energy consumed by the normal transmission is also more. But in conditional transmission the frequency of transmission is less since the data will be transmitted only when the value is not within acceptable level. So the energy consumed by conditional transmission is very less. Simulation result has been taken to compare with normal transmission.

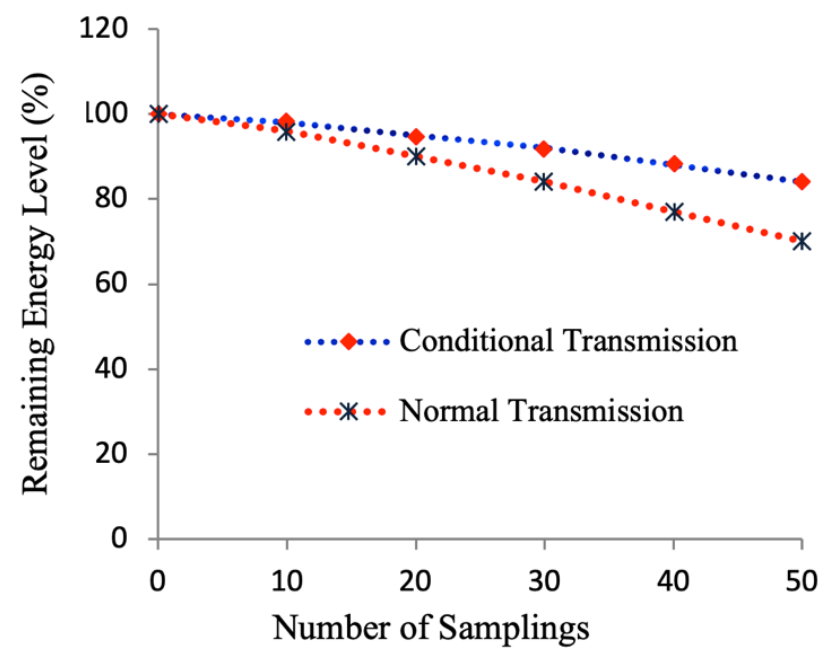

Fig. 3. Energy level comparison with respect to number of samplings 


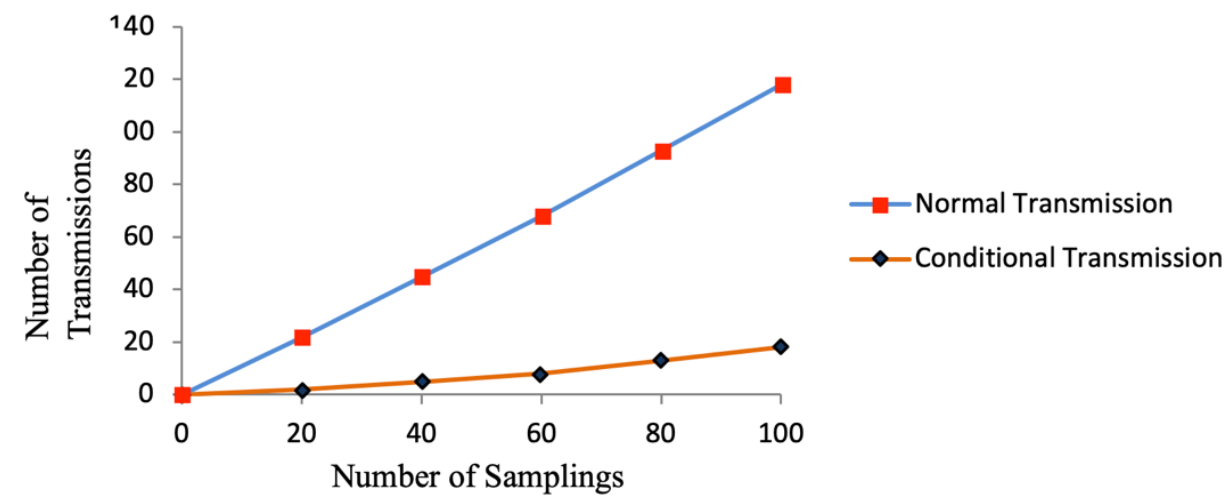

Fig. 4. Number of transmissions based on number of samplings

When we take number of transmissions based on samplings, in normal transmission there will be equal number of transmissions as that of number of samplings, but in conditional transmission the number of transmission greatly reduced since it send only critical values. Based on this scenario the energy consumption will be calculated.

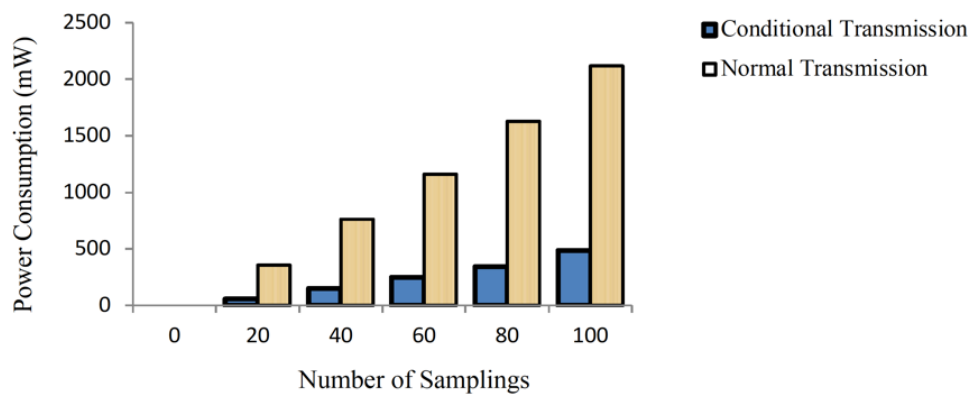

Fig. 5. Representation of energy consumption by conditional and normal transmission

Simulation result from figure 5 undoubtedly proves that the power consumed by normal transmission is more than conditional transmission. There are 100 samplings are taken for the simulation. Since conditional transmission took only few transmissions to transmit the data it consumes less power. In turn the energy consumed by the proposed method is very less.

\section{Conclusion and Future Works}

Sensors are very useful in monitoring human health remotely. At the same time it has few issues like lifetime of node, reliability and so on. When the power utilized properly then the lifetime of the node can be increased. Our proposed approach conditional transmission technique along with on demand sleep awake algorithm helps to reduce the energy consumption and in turn increases the lifetime. Reliability is also very much essential. So in future this research can be focused reliability as well. 


\section{$7 \quad$ References}

[1] H. Karl and A. Willig, "Protocols and Architectures for Wireless Sensor Networks,"John Wiley \& Sons, Hoboken, 2005. https://doi.org/10.1002/0470095121

[2] J. L. Hill, "System Architecture for Wireless Sensor Networks," University of California, Berkeley, 2003.

[3] Muhammad asghar khan, Asfandyar Khan, Said Khalid Shah, Azween Abdullah,” An Energy Efficient Color Based Topology Control Algorithm for Wireless Sensor Networks", Scientific Research, Wireless Sensor Networks, 2013, 5, 1-7. https://doi.org/10. 4236/wsn.2013.51001

[4] Giuseppe Anastasi, Marco Conti, Mario Di Francesco," Extending the Lifetime of Wireless Sensor

[5] Networks through Adaptive Sleep", IEEE Transactions.

[6] Subhash Dhar Dwivedi, Praveen Kaushik," Energy Efficient Routing Algorithm with sleep scheduling in Wireless Sensor Network", Subhash Dhar Dwivedi et al, / (IJCSIT) International Journal of Computer Science and Information Technologies, Vol. 3 (3) , 2012,4350 4353.

[7] Amit Sharma, Kshitij Shinghal, Neelam Srivastava, Raghuvir Singh,” Energy Management for Wireless Sensor Network Nodes", International Journal of Advances in Engineering \& Technology, Vol. 1, Mar 2011. https://doi.org/10.7323/ijaet/v1 iss1 2

[8] Zahra Rezaei , Shima Mobininejad," Energy Saving in Wireless Sensor Networks", International Journal of Computer Science \& Engineering Survey (IJCSES) Vol.3, No.1, February 2012.

[9] T. Shah, N. Javaid, T. N. Qureshi," Energy Efficient Sleep Awake Aware (EESAA) Intelligent Sensor Network Routing Protocol", arXiv: 1212.4106v1 [cs.NI] 17 Dec 2012.

[10] Huan-Chao Keh*, Ying-Hong Wang, Kun-Yi Lin and Cheng-Che Lin,'Power Saving Mechanism with Optimal Sleep Control in Wireless Sensor Networks", Tamkang Journal of Science and Engineering, Vol. 14, No. 3, pp. 235243 (2011).

[11] Luiz H.A. Correia, Daniel F. Macedo, Aldri L. dos Santos, Antonio A.F. Loureiro, Jose ' Marcos S. Nogueira," Transmission power control techniques for wireless sensor networks",

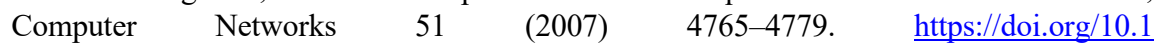
016/j.comnet.2007.07.008

[12] Samaneh Movassaghi, Mehran Abolhasan, Justin $\quad$ Lipman, David Smith," Wireless Body Area Networks: A Survey", IEEE Communications Surveys \& Tutorials, January 2014.

[13] Aravind Kailas," Power Allocation Strategies to Minimize Energy Consumption in Wireless Body Area Networks", 33rd Annual International Conference of the IEEE EMBS, Boston, Massachusetts USA, August 30 - September 3, 2011.

[14] Aan Jelli Priana, Herman Tolle, Ismiarta Aknuranda, "User Experience Design of Stroke Patient Communications Using Mobile Finger (MOFI) Communication Board With User Center Design Approach", International Journal of Interactive Mobile Technologies, Vol 2, No 2, 2018. https://doi.org/10.3991/ijim.v12i2.7937

[15] Orawit Thinnukool(!) , Pattaraporn Khuwuthyakorn, Purida Wientong," Non-Prescription Medicine Mobile Healthcare Application: Smartphone-Based Software Design and Development Review", International Journal of Interactive Mobile Technologies, Vol 11, No 5, 2017. https://doi.org/10.3991/ijim.v11i5.7123.

[16] Orawit Thinnukool(!), Pattaraporn Khuwuthyakorn, Purida Wientong," Pharmacy Assistant Mobile Application (PAMA): Development and Reviews", International Journal of 
Short Paper-Increasing Lifetime through Sleep Awake and Conditional Transmission...

Interactive Mobile Technologies, Vol 11, No 3, 2017. https://doi.org/10. 3991/ijim.v11i3.6757.

\section{Authors}

Karthik. $\mathbf{J}$ is a Research Scholar in department of Computer Science and Engineering, St Peters Institute of Higher Education and Research, Avadi, Chennai, Tamil Nadu, India. His Research Interest is Wireless Body Area Sensor Networks. His mail id is karthikvalavan@gmail.com.

Dr. A. Rajesh is working as a Professor and Head of Institution, C. Abdul Hakeem College of Engineering and Technology, Melvisharam, Vellore, Tamil Nadu, India. His Research Interest is Wireless Sensor Networks and Image Processing.

Article submitted 15 November 2018. Resubmitted 08 January 2019. Final acceptance 16 January 2019. Final version published as submitted by the authors. 